\title{
MONODROMY OF CONSTANT MEAN CURVATURE SURFACE IN HYPERBOLIC SPACE*
}

\author{
GIAN PIETRO PIROLA ${ }^{\dagger}$
}

\begin{abstract}
In this paper we give a global version of the Bryant representation of surfaces of constant mean curvature one (cmc-1 surfaces) in hyperbolic space. This allows to set the associated non-abelian period problem in the framework of flat unitary vector bundles on Riemann surfaces. We use this machinery to prove the existence of certain cmc-1 surfaces having prescribed global monodromy.
\end{abstract}

Key words. Monodromy, constant curvature, hyperbolic space

AMS subject classifications. 58E15

Introduction. The local theory of surfaces of constant mean curvature one (cmc1 surfaces) in hyperbolic space is equivalent to the local theory of minimal surfaces in Euclidean space. If this was originally the main reason for their study (but see also [4]), the interest in the topic was renewed by the fundamental work of Robert Bryant [2]. The "Bryant-Weierstrass" formula (see also [3] for the analysis of an earlier formulation) allows to represent these surfaces by holomorphic mappings. To explain this, let $S L(2, \mathbb{C})$ be the special linear group and $S U(2)$ the special unitary group. We identify the quotient $S L(2, \mathbb{C}) / S U(2)$ with the hyperbolic 3 -space $\mathcal{H}^{3}$. Letting $\pi: S L(2, \mathbb{C}) \rightarrow \mathcal{H}^{3}$ be the quotient then [2] a simply connected cmc-1 surface in $\mathcal{H}^{3}$ arises as $S=f(U)$, where $U$ is an open set of the complex plane, $f=\pi \cdot g$ and $g: U \rightarrow S L(2, \mathbb{C})$ is a holomorphic map. In other words, the entries of the matrix

$$
g(z)=\left(\begin{array}{ll}
a(z) & b(z) \\
c(z) & d(z)
\end{array}\right)
$$

are holomorphic; moreover they satisfy the Bryant conditions:

$$
\operatorname{det} g(z)=1, \quad \operatorname{det} g^{\prime}(z)=\operatorname{det}\left(\begin{array}{ll}
a^{\prime}(z) & b^{\prime}(z) \\
c^{\prime}(z) & d^{\prime}(z)
\end{array}\right)=0 .
$$

The global theory of cmc-1 surfaces substantially differs from the theory of minimal surfaces in flat spaces. In fact, since $S U(2)$ is non abelian, the period problem cannot be solved by classical potential theory. In this paper we set the BryantWeierstrass representation formula in the framework of unitary bundles on Riemann surfaces. The period problem becomes then equivalent to the existence of suitable holomorphic sections of a flat bundle. The holonomy of the flat connection encodes the monodromy data. To explain this, let $X$ be a Riemann surface and $f: X \rightarrow \mathcal{H}^{3}$ be a conformal immersion such that $f(X)$ is a cmc- 1 surface. Then there are a rank 2 vector bundle $F$, with flat $S U(2)$ connection $\nabla$, and two holomorphic sections of $F$, $s_{1}$ and $s_{2}$, satisfying:

$$
\operatorname{det}\left(s_{1}, s_{2}\right)=1, \operatorname{det}\left(\nabla s_{1}, \nabla s_{2}\right)=0
$$

\footnotetext{
*Received November 8, 2005; accepted for publication November 20, 2006.

†Dipartimento di Matematica, Università di Pavia, via Ferrata 1, 27100 Pavia, Italy (gianpietro. pirola@unipv.it). Partially supported by 1) PRIN 2005 "Spazi di moduli e teorie di Lie"; 2) INDAM; 3) Far 2006 (PV): Varietà algebriche, calcolo algebrico, grafi orientati e topologici.
} 
With respect to a unitary basis for the space of harmonic sections of $F$, the equations in (2) become the Bryant conditions (1). Fix a point $x \in X$ and let $\Pi_{1}(X, x)$ denote the fundamental group of $X$ at $x$. The monodromy map associated to $f$ is a homomorphism:

$$
m(f): \Pi_{1}(X, x) \rightarrow S U(2),
$$

which is the holonomy of $\nabla$. Conversely, two holomorphic sections of a flat $S U(2)-$ bundle on $X$ that satisfy the equations (2) define a cmc-1 surface in $\mathcal{H}^{3}$.

In the case of an algebraic complex curve, the flat unitary bundles are equivalent to certain stable parabolic holomorphic vector bundles. This allows, via a RiemannRoch type theorem, to prove the existence of surfaces with fixed monodromy. More precisely, let $Y$ be a compact Riemann surface and $H \subset Y$ be any finite subset, possibly empty. The result given in 4.3 .4 implies (see the end of section 4) the following:

EXIstence TheOREM. Let $m: \Pi_{1}(Y \backslash H) \rightarrow S U(2)$ be a group homomorphism. Assume that the image of $m$ is not an abelian group. Then there is a finite set $D \subset Y$ and an immersion $f: Y \backslash(D \cup H) \rightarrow \mathcal{H}^{3}$ such that $f(Y \backslash(D \cup H))$ is a cmc-1 surface and $m(f): \Pi_{1}(Y \backslash(D \cup H)) \rightarrow S U(2)$ factors through $m$. That is $m(f)=m j_{*}$ where

$$
j_{*}: \Pi_{1}(Y \backslash(D \cup H)) \rightarrow \Pi_{1}(Y \backslash H)
$$

is induced by the inclusion.

Moreover in 4.3.4 the sections are meromorphic at $D \cup H$, the ends of $f$. The study of meromorphic sections satisfying the Bryant conditions (2) turns out to be quite interesting. Our proof of the existence result relies on a homogenization procedure. This allows to use intersection theory in projective spaces. We note we are not able to prove that our surfaces are complete: if we complete them, we are no longer able to prove they are immersed. This problem should be clarified by an analysis of the moduli space parametrizing sections that satisfy Bryant conditions. In contrast with the Euclidean minimal surfaces case (see [11]), an infinitesimal useful description has not yet been found.

The paper is organized as follows. In section 1, starting from simple consideration on complex Lie groups, we obtain the global version of the Bryant-Weierstrass representation 1.4.3. In section 2 we analyze the case of a punctured disk and the induced local parabolic structure. In 2.2.2 we give a simple, but important, remark on the Laurent series of our sections. In section 3 we recall the correspondence between stable and flat $S U(2)$ bundle on a smooth complex algebraic curve. We remark the result of lemma 3.2.3. It provides a properness result in the case of irreducible connections. In section 4 , all previous results are collected to prove our main Theorem 4.3.4. A more general setting is considered in section 5, which is in certain sense a complementary section. We use the unifying language of Higgs fields to consider both the surfaces introduced in [10] and the periodic cmc-1 case. This allows to formulate a general non-abelian period problem (see 5.1.1).

It is a great pleasure to thank Maasaki Umehara for his precious advice. I am really grateful to him. I would like to thank Enrico Schlesinger, who read the manuscript and suggested many improvements. 


\section{Bryant representation.}

1.1. Lie groups and flat structures. Let $X$ be a smooth connected Riemann surface and $\mathcal{O}_{X}$ be the holomorphic structure sheaf of $X$. Let $G$ be a complex Lie group and let $g$ be its Lie algebra. We denote by $J: g \rightarrow g$ the map induced by the complex structure. Let $H$ be a closed (Lie) subgroup of $G$ with Lie algebra $h$. We shall assume $H$ transverse to the complex structure of $G$, that is:

$$
J h \cap h=0_{g} .
$$

Letting $\pi: G \rightarrow G / H$ be the quotient, we consider maps $f: X \rightarrow G / H$. We say that $f$ is $h$-liftable if there are local holomorphic liftings of $f$ to $G$. This means that there exist an open covering $\left\{U_{\alpha}\right\}_{\alpha \in I}$ of $X$ and holomorphic maps:

$$
g_{\alpha}: U_{\alpha} \rightarrow G
$$

such that $\pi g_{\alpha}=f \mid U_{\alpha}$. For $\alpha$ and $\beta \in I$ we define

$$
g_{\alpha \beta}=g_{\alpha}^{-1} \cdot g_{\beta}: U_{\alpha} \cap U_{\beta} \rightarrow H \subset G .
$$

Since $g_{\alpha \beta}$ is holomorphic and $H$ is transverse to the complex structure, the $g_{\alpha \beta}$ are locally constant and therefore define a $H$-principal flat bundle on $X$. If a point $p$ of $X$ is fixed, loops restriction induces the monodromy homomorphism :

$$
m: \Pi(X, p) \rightarrow H \subset G
$$

If $V$ is a complex vector bundle and $\rho: G \rightarrow G L(V, \mathbb{C})$ is a complex linear representation, the $\rho\left(g_{\alpha \beta}\right)$ are transition functions for a flat complex vector bundle $F$ on $X$. The structure group of $F$ reduces to $\rho(H)$. Then $F$ has a natural holomorphic structure and the composition

$$
\theta=\rho \cdot m: \Pi(X, p) \rightarrow \rho(H) \subset G L(V)
$$

is the associated monodromy representation.

1.2. Special and unitary group. Let $M(n)$ denote the vector space of square complex matrices of order $n$. In the sequel, unless specified differently, $G$ and $H$ will denote respectively the special linear group $G=S L(n, \mathbb{C})=\{g \in M(n): \operatorname{det}(g)=1\}$, and the special unitary group $H=S U(n)=\left\{g \in S L(n, \mathbb{C}): g \cdot g^{*}=e\right\}$, where $e \in M(n)$ is the identity matrix and $g^{*}={ }^{t} \bar{g}$ is the adjoint of $g$. Clearly, $H$ is transverse to the complex structure of $G: h=s u(n)$. By the spectral theorem we identify the quotient $S L(n, \mathbb{C}) / S U(n)$ with

$$
L(n)=\left\{g \in S L(n, \mathbb{C}): g=g^{*}, g>0\right\}
$$

so that

$$
\pi(g)=g \cdot g^{*}
$$

Let $f: X \rightarrow L(n)$ be a $h$-liftable map and let $\left\{g_{\alpha \beta}\right\}$ be the $S U(n)$-cocycle defined in (5). Let $W=\mathbb{C}^{n}$ be the standard representation of $S U(n)$ and $F$ be the associated flat complex vector bundle on $X$. Note that $F$ is a rank $n$ holomorphic vector bundle on $X$ with trivial determinant. The natural hermitian product on $F$ will be denoted by $<,>$. For a fixed $v \in W$, the collection $\left\{g_{\beta}^{-1} v\right\}_{\beta \in I}$ (see formula 
4) defines a holomorphic section of $F$. Letting $H^{0}(X, F)$ denote the vector space of the holomorphic sections of $F$, we thus obtain an inclusion $j: W \rightarrow H^{0}(X, F)$. We let $\left\{v_{i}\right\}_{i=1, \ldots, n}$ be the canonical basis of $W$, and set $j\left(v_{i}\right)=e_{i}$. Under the isomorphism $\operatorname{det}(F)=\mathcal{O}_{X}$ we have:

$$
e_{1} \wedge \ldots \wedge e_{n}=1
$$

If we denote by $F_{x}$ the fiber of $F$ over $x \in X$ the $e_{i}(x)$ give a $S L(n, \mathbb{C})$ basis of $F_{x}$ at every point $x \in X$.

Definition 1.2.1. A holomorphic frame $\left\{e_{i}\right\}_{i=1, \ldots, n}$ satisfying (7) will be called a special frame of $F$.

To any $h$-liftable map $f: X \rightarrow L(n)=S l(n, \mathbb{C}) / S U(n)$ is associated a special frame $e_{1} \ldots e_{n}$ of a $S U(n)$ flat vector bundle $F$. Conversely, if $F$ is a $S U(n, \mathbb{C})$ flat vector bundle on $X$ and $e_{1} \ldots e_{n}$ is a special frame of $F$, for every $x \in X$ we define

$$
A(x)=\left(e_{1}(x), \ldots, e_{n}(x)\right) \in \operatorname{Hom}\left(\mathbb{C}^{n}, F_{x}\right) .
$$

This gives a holomorphic section $A \in \operatorname{Hom}\left(\mathbb{C}^{n}, F\right)$. On the other hand, the adjoint $A^{*}(x)$ :

$$
<A(x) v, w>{ }_{x}=<v, A^{*}(x) w>,
$$

provides an antiholomorphic section $A^{*}$ of $\operatorname{Hom}\left(F, \mathbb{C}^{n}\right)$. Then

$$
f(x)=A(x) \cdot A^{*}(x) \in L(n)=S l(n, \mathbb{C}) / S U(n)
$$

defines a $h$-liftable map $f: X \rightarrow S l(n, \mathbb{C}) / S U(n)$, the holomorphic local lifting of $f$ being $A(x)$ written in local coordinates.

Definition 1.2.2. Two special frames $\left\{e_{i}\right\}_{i=1, \ldots, n}$ and $\left\{f_{i}\right\}_{i=1, \ldots, n}$ of $F$ will be said equivalent if there is $U \in S U(n)$ such that $U e_{i}=f_{i} \quad i=1 \ldots, n$. have:

Special equivalent frames correspond to the same $h$-liftable mapping $f$, thus we

Proposition 1.2.3. There is a one to one correspondence between h-liftable maps

$$
f: X \rightarrow S L(n, \mathbb{C}) / S U(n)
$$

and equivalence classes of special frames of $S U(n)$-flat vector bundles of $X$.

Proof. Clear.

The monodromy (6) gives a map $\theta: \Pi(X, p) \rightarrow S U(n)$. The group

$$
G_{\theta}=i m(\theta)
$$

is well defined up to conjugation and determines the flat bundle $F$.

Definition 1.2.4. The flat bundle $F$ is said reducible (resp. irreducible) if the corresponding representation $\theta$ of the fundamental group is reducible (resp. irreducible).

REMARK 1.2.5. There are many Lie subgroups $H \subset S L(n, \mathbb{C})$ transverse to the complex structure, for instance $S L(n, \mathbb{R})$. For our purposes, however, it is useful to 
assume $h=s u(n)$, that is, the connected component $H_{e}$ of $H$ is the special unitary group: $H_{e}=S U(n)$. In this case $K=H / H_{e}$ is a discrete group acting on $L(n)$. We have $L(n) / K=G / H$. If $f: X \rightarrow G / H$ is as before and $K$ acts freely on $L(n)$, by taking a suitable covering $\tilde{X} \rightarrow X$, we may define a periodic lifting (see also section 5) $\tilde{f}: \tilde{X} \rightarrow L(n)$.

1.3. Flat connections. We fix a flat $S U(n)$ vector bundle $F$ on $X$ with hermitian metric $<,>$. Denote by $C^{\infty}(F)$ the sheaf of smooth sections of $F$. Let $A^{k}(F)=C^{\infty}\left(F \otimes \Omega_{X}^{k}\right)$ be the sheaf of $k$-forms on $F$. Using the complex structure on $X$ we can also consider the space $A^{p, q}(F)$ of $p, q$ forms on $X$ ([5]). Let $\nabla: A^{k}(F) \rightarrow A^{k+1}(F)$ (see [9]) be the associated unitary flat connection

$$
\nabla^{2}=0
$$

which preserves the metric

$$
d<s, t>=<\nabla s, t>+<s, \nabla t>
$$

and the complex structure:

$$
\nabla^{\prime \prime}=\bar{\partial}
$$

Here $\nabla^{\prime \prime}$ denotes the composition of $\nabla: C^{\infty}(F) \rightarrow A^{1}(F)$ and the projection $A^{1}(F) \rightarrow$ $A^{0,1}(F)$. Let

$$
\operatorname{ker} \nabla \subset C^{\infty}(F)
$$

be the sheaf of harmonic sections of $F$. We have $\operatorname{ker} \nabla \subset \operatorname{ker} \nabla^{\prime \prime}$. We identify as usual $F$ with the sheaf ker $\nabla^{\prime \prime}$ of holomorphic sections of $F$.

REMARK 1.3.1. The holonomy of $\nabla$ associated to $F$ is (up conjugation) the group $G_{\theta}$ (see 8). We say that $\nabla$ is irreducible if the monodromy representation 1.2.4 is irreducible.

1.4. Bryant condition. We now set $G=S L(2, \mathbb{C})$ and $H=S U(2)$. The quotient $L(2)=G / H$ can be identified with the hyperbolic 3 -space $\mathcal{H}^{3}$. By $1.2 .3 \mathrm{~h}$ liftable maps $f: X \rightarrow \mathcal{H}^{3}$ correspond to special frames of $S U(2)$ vector bundles on $X$. We fix a flat $S U(2)$ vector bundle $F$ on $X$, and denote by $\nabla$ its connection. We remark that $\nabla$, (see 1.3.1), is reducible if and only if $G_{\theta}$ is abelian and hence, up to conjugation, $G_{\theta}$ is contained in the subgroup of diagonal matrices.

If $s$ is a holomorphic section of $F, s \in H^{0}(X, F)$, then $\nabla s$ is a holomorphic section of $F \otimes \omega_{X}, \nabla s \in H^{0}\left(X, F \otimes \omega_{X}\right)$. Here $\omega_{X}$ denotes the sheaf of holomorphic differentials on $X$. Since $\operatorname{det} F$ is trivial, $\operatorname{det}\left(F \otimes \omega_{X}\right)=\omega_{X}^{2}$ is the sheaf of holomorphic quadratic differentials of $X$. If $s \in H^{0}(X, F)$ and $t \in H^{0}(X, F)$ we define:

$$
\Omega(s, t)=\nabla s \wedge \nabla t \in H^{0}\left(X, \omega_{X}^{2}\right) .
$$

The vanishing of the above differential (9) has an important geometric meaning (see [2]). Recall that a special frame (see 1.2.1) of $F$ is a pair of holomorphic sections $s$ and $t$ such that

$$
s \wedge t=1
$$


Definition 1.4.1. We say the special frame $(s, t)$ of $F$ satisfies the Bryant condition if $\nabla s \wedge \nabla t=0$. In this case $(s, t)$ will be called a $\mathcal{B}$-frame.

Let $(s, t)$ be $\mathcal{B}$-frame of $F$. Fix a point $\bar{x} \in X$ and let $\{U(\bar{x}), z\}$ be a simply connected open coordinate neighborhood of $\bar{x}$. Let $z: U(\bar{x}) \rightarrow \mathbb{C}$ be the coordinate map. We can find, by parallel transport, a harmonic unitary frame $h_{1}, h_{2}$ of $F$, $\nabla h_{i}=0$, on $U(\bar{x})$ such that $h_{1} \wedge h_{2}=1$. We may write:

$$
s=a(z) h_{1}+c(z) h_{1} ; \quad t=b(z) h_{1}+d(z) h_{1},
$$

hence

$$
\nabla s=\left(a^{\prime}(z) h_{1}+c^{\prime}(z) h_{2}\right) d z ; \quad \nabla t=\left(b^{\prime}(z) h_{1}+d^{\prime}(z) h_{2}\right) d z .
$$

Here $a, b, c$ and $d$ are holomorphic functions. Define the matrix

$$
A(z)=\left(\begin{array}{ll}
a(z) & b(z) \\
c(z) & d(z)
\end{array}\right)
$$

and note $\operatorname{det} A(z)=1$. Taking derivative we may write the Bryant condition as

$$
\operatorname{det} A^{\prime}(z)=\operatorname{det}\left(\begin{array}{ll}
a^{\prime}(z) & b^{\prime}(z) \\
c^{\prime}(z) & d^{\prime}(z)
\end{array}\right)=0
$$

Let $f: U(x) \rightarrow \mathcal{H}^{3}($ see 1.2 .3$)$

$$
f(z)=A(z) \cdot A^{*}(z),
$$

be the associated map. Assume that $f$ is non-constant, then following [2] (see also [13], [14] and the H. Rosenberg contribution in [6]), we observe that (10) gives the Bryant-Weierstrass representation of the constant mean curvature one (cmc-1) surface $f(X) \subset \mathcal{H}^{3}$. We have proven:

Proposition 1.4.2. The maps $f: X \rightarrow \mathcal{H}^{3}$ such that $f(X)$ is a (branched) cmc-1 surface are in one to one correspondence with equivalence classes (see 1.2.2) of non-trivial $\mathcal{B}$-frames 1.4.1.

One verifies that, if $(s, t)$ is a $\mathcal{B}$-frame, then $f: X \rightarrow \mathcal{H}^{3}$ is an immersion if and only if all the local holomorphic lifting $g_{\alpha}: U_{\alpha} \rightarrow S L(2, \mathbb{C})$ are immersions (see [6]). It follows then that $x$ is a branch point if and only if $A^{\prime}(z(x))=0$ (see 10). Therefore $f$ is an immersion if and only if the set of branch points of $f$

$$
Z(f)=\{x \in X: \nabla s(x)=\nabla t(x)=0\}
$$

is empty. We finally state the following (compare with [2]):

Proposition 1.4.3. Global Bryant-Weierstrass representation Let $M \subset$ $\mathcal{H}^{3}$ be a cmc-1 immersed surface. Then $M=f(X)$ where $X$ is a Riemann surface, and $f: X \rightarrow \mathcal{H}^{3}$ is the h-liftable map associated to a pair of sections $s, t$ of a $S U(2)$ flat bundle $F$ such that

a) $\bar{\partial} s=\bar{\partial} t=0$ (holomorphicity);

b) $s \wedge t=1$ (special-frame);

c) $\nabla s \wedge \nabla t=0$ (Bryant condition);

d) $Z=\{x \in X: \nabla s(x)=\nabla t(x)=0\}=\emptyset$ (immersion). 
2. The punctured disk: the end of a surface. Let $\Delta=\{z \in \mathbb{C}: \mid z<1\}$ be the unit disk and denote by $O$ the origin of $\Delta$. Set $\Delta^{*}=\Delta-\{O\}$. We will recall the classical description of the $S U(2)$ connections of $\Delta^{*}$ which will be used in our setting.

2.1. Singular connection on $\Delta$. Since the fundamental group of $\Delta^{*}$ is cyclic, any $S U(2)$ bundle over $\Delta^{*}$ is reducible (see 1.2.4 and 1.3.1) and the holonomy map can be described by a single matrix $T(a)$ :

$$
T(\alpha)=\left(\begin{array}{cc}
\exp (\pi i \alpha) & 0 \\
0 & \exp (-\pi i \alpha)
\end{array}\right)
$$

where $\alpha \in \mathbb{R}, 0 \leq \alpha<1$. The holonomy group is $G_{\alpha}=\left\{T(\alpha)^{n}: n \in \mathbb{Z}\right\}$. To construct a flat bundle with $G_{\alpha}$-holonomy, take the trivial bundle

$$
\mathbb{C}^{2} \times \Delta \rightarrow \Delta
$$

Then we define the singular connection:

$$
\bar{\nabla}_{\alpha}=d-\left(\begin{array}{cc}
\alpha & 0 \\
0 & -\alpha
\end{array}\right) \frac{d z}{z}
$$

that is,

$$
\bar{\nabla}_{\alpha}(f, g)=\left(d f-\alpha \frac{f}{z} d z, d g+\alpha \frac{g}{z} d z\right) .
$$

Let $\mathcal{O}_{\Delta}(n O)$ be the sheaf of meromorphic function on $\Delta$ that are holomorphic on $\Delta^{*}$ and have at most at most a pole of order $n$ at the origin $O$ of $\Delta$. We have

$$
\bar{\nabla}: \mathcal{O}_{\Delta}^{2} \rightarrow \mathcal{O}_{\Delta}^{2}(O) \otimes \omega_{\Delta}
$$

Its residue

$$
\operatorname{res} \bar{\nabla}_{\alpha}=\Gamma_{\alpha}=\left(\begin{array}{cc}
\alpha & 0 \\
0 & -\alpha
\end{array}\right)
$$

is an operator on $\mathbb{C}^{2}$, the fiber of $O$, having eigenvalues $\alpha$ and $-\alpha$, and, if $0<\alpha<1$, eigenspaces $L_{\alpha}=\{(z, 0): z \in \mathbb{C}\}$ and $L_{-\alpha}=\{(0, z): z \in \mathbb{C}\}$. Let $V=\left.\mathbb{C}^{2}\right|_{\Delta^{*}}$ be the restriction on $\Delta^{*}$ we set

$$
\nabla_{\alpha}=\left.\bar{\nabla}_{\alpha}\right|_{\Delta^{*}}
$$

Note that $\nabla_{\alpha}$ is a flat connection on $V$. The matrix

$$
H(\alpha)=\left(\begin{array}{cc}
w^{\alpha} & 0 \\
0 & w^{-\alpha}
\end{array}\right) w=z \cdot \bar{z}=x^{2}+y^{2}, z=x+i y,
$$

defines an hermitian product $<,>_{\alpha}$ on $V$ compatible with $\nabla_{\alpha}$. The holonomy group of $\nabla_{\alpha}$ is $G_{\alpha}$. We remark that the metric $<,>_{\alpha}$ extends continuously by 0 on $L_{\alpha}$ and gives a natural filtration

$$
0 \subsetneq L_{\alpha} \subsetneq \mathbb{C}_{O}^{2}
$$

We have: 
Proposition 2.1.1. The connection $\nabla_{\alpha}$ is a SU(2) flat connection with holonomy $G_{\alpha}$.

Proof. One has $\nabla_{\alpha}^{2}=0, \nabla_{\alpha}^{\prime \prime}=\bar{\partial}$ and $\nabla_{\alpha}$ is compatible with $<,>_{\alpha} . \square$

Remark 2.1.2. Set $\widetilde{d}=\wedge^{2} \bar{\nabla}$. Then $\widetilde{d}$ defines the trivial connection on $\operatorname{det} E$. In fact, let $v_{1}=(0,1), v_{2}=(1,0)$ be the constant sections, $u=v_{1} \wedge v_{2}$ and fix a function b. We have

$$
\begin{gathered}
\widetilde{d}(b \cdot u)=d\left(b\left(v_{1} \wedge v_{2}\right)\right)+b\left(\bar{\nabla}_{\alpha}\left(v_{1}\right) \wedge v_{2}+v_{1} \wedge \bar{\nabla}_{\alpha}\left(v_{2}\right)\right)= \\
(d b) \cdot\left(v_{1} \wedge v_{2}\right)+b \frac{\alpha d z}{z}\left(-v_{1} \wedge v_{2}+v_{1} \wedge v_{2}\right)=(d b) \cdot u,
\end{gathered}
$$

that is, $\widetilde{d}=d$.

If $\{F, \nabla\}$ is a $S U(2)$ flat bundle on $\Delta^{*}$ then it is isomorphic to $\left\{V, \nabla_{\alpha}\right\}$ for some $\alpha, 0 \leq \alpha<1$. Therefore there is a natural logarithmic extension $\{E, \bar{\nabla}\}$ of $\{F, \nabla\}$ to $\Delta$ :

$$
\bar{\nabla}: E \rightarrow E(O) \otimes \omega_{\Delta},
$$

where $E$ is isomorphic to the trivial bundle $\mathbb{C}^{2}$ and $E(O)=E \otimes \mathcal{O}_{\Delta}(O)$. Moreover, if $E_{O}$ is the fiber of $E$ at $O, \Gamma=\operatorname{res} \bar{\nabla} \in \operatorname{Hom}\left(E_{0}, E_{0}\right)$ has eigenvalue $\alpha$ and $-\alpha$. If the holonomy is non-trivial, i.e. $\alpha>0$, let $W=\operatorname{ker}(\Gamma-\alpha I d)$ where $i d$ is the identity. The hermitian product on $E$ extends by 0 on all of $W$. We have a filtration $E_{0} \supsetneq W \supsetneq 0$.

Definition 2.1.3. Suppose $\{F, \nabla\}$ is a $S U(2)$ flat bundle $\Delta^{*}$ with extension $\{E, \bar{\nabla}\} \simeq\left\{\mathbb{C}^{2}, \bar{\nabla}_{\alpha}\right\}, 0 \leq \alpha<1$. If $\{F, \nabla\}$ has non trivial holonomy, i.e. $\alpha>0$, the associated local parabolic structure on $E$ is given by:

i) the weight $\alpha \in] 0,1[\subset \mathbb{R}$;

ii) the filtration $E_{0} \supsetneq W \supset 0$ where $W=\operatorname{ker}(\Gamma-\alpha i d)$ and $\Gamma=\operatorname{res} \bar{\nabla}_{\alpha}$.

2.2. Bryant condition on $\Delta^{*}$. We write Bryant condition for sections of $\left\{V, \nabla_{\alpha}\right\}$. Let $s$ and $t$ be holomorphic sections of $V$. Laurent expansion allows to write $s=(a(z), b(z))$ and $t=(c(z), d(z))$ as singular sections of $\mathbb{C}^{2}$. Assume $s \wedge t=1$, i.e. $a d-b c=1$. Let $f: \Delta^{*} \rightarrow \mathcal{H}^{3}$ be the $h$-liftable map associated to this special frame 1.2.3.

Definition 2.2.1. We say the frame $(s, t)$ has finite type if $s$ and $t$ extend meromorphically to $\Delta$. The order of $(s, t)$ is the smallest integer $n \geq 0$ such that $z^{n} s$ and $z^{n} t$ are holomorphic. We also say the h-liftable associated map (or cmc-1 surface immersion) has order $n$.

Denote by $\Omega_{\alpha}(s, t)$ - compare formula (9) above - the singular extension of $\nabla_{\alpha} s \wedge \nabla_{\alpha} t$ to $\Delta$ :

$$
\Omega_{\alpha}(s, t)=\bar{\nabla}_{\alpha} s \wedge \bar{\nabla}_{\alpha} t=\operatorname{det}\left[\left(\begin{array}{ll}
a^{\prime} & b^{\prime} \\
c^{\prime} & d^{\prime}
\end{array}\right)-\alpha \frac{1}{z}\left(\begin{array}{ll}
a & -b \\
c & -d
\end{array}\right)\right](d z)^{2} .
$$

Let

$$
H^{0}\left(\Delta, \omega_{\Delta}^{2}(m)\right)=H^{0}\left(\Delta, \omega_{\Delta}^{2} \otimes \mathcal{O}_{\Delta}(m O)\right)
$$


be the space of holomorphic quadratic differentials on $\Delta$ having a pole of order at most $m$ at $O$. If $(s, t)$ has finite type of order $n$, then $\Omega_{\alpha}(s, t)=\bar{\nabla}_{\alpha} s \wedge \bar{\nabla}_{\alpha} t \in$ $H^{0}\left(\Delta, \omega_{\Delta}^{2}(2 n+2)\right)$.

The following more precise result will play a role in our existence result:

Lemma 2.2.2. Assume $s, t \in H^{0}\left(\Delta, \mathcal{O}_{\Delta}(n O)\right.$ ), and $s \wedge t=1$ (or else $s \wedge t$ holomorphic at $O)$. We have

1. $\Omega_{\alpha}(s, t) \in H^{0}\left(\Delta, \omega_{\Delta}^{2}(2 n+1)\right)$ if $0<\alpha<1$;

2. $\Omega_{0}(s, t) \in H^{0}\left(\Delta, \omega_{\Delta}^{2}(2 n)\right)$.

Proof. Write the condition that $s \wedge t$ is holomorphic on the Laurent series of $s$ and $t$. Then compare with the Laurent series of equation 13. $\square$

Now assume that $(s, t)$ is a Bryant frame, that is, $s \wedge t=1$ and $\Omega_{\alpha}(s, t)=0$ 1.4.1. We obtain the:

Bryant equations for the punctured disk:

$$
\left\{\begin{array}{l}
a d-b c=1 \\
z^{2}\left(a^{\prime} d^{\prime}-b^{\prime} c^{\prime}\right)+z \alpha\left(a d^{\prime}-a^{\prime} d-b^{\prime} c+b c^{\prime}\right)-\alpha^{2}=0 .
\end{array}\right.
$$

3. Flat bundles on algebraic complex curves. Non-abelian Hodge-theory gives correspondences between representations of the fundamental group of algebraic (or compact Kähler) varieties, that is, flat vector bundles, and algebraic objects: (parabolic) stable (Higgs) bundles. We will only describe the $S U(2)$ case on algebraic curves.

3.1. Parabolic and stable bundles. Let $Y$ be a compact Riemann surface. A real divisor of $Y$ is a finite combination $S=\sum a_{i} P_{i}, a_{i} \in \mathbb{R}, P_{i} \in Y$. We will always assume that $P_{i} \neq P_{j}$ if $i \neq j$. We say that $S$ is effective if $a_{i} \geq 0$ for all $i$. The support of $S$ is the divisor

$$
\operatorname{supp}(S)=\sum P_{i}: a_{i} \neq 0 .
$$

A divisor $D$ is simple if $D=\operatorname{supp}(D)$. We identify as usual the set $\cup_{i=1}^{n}\left\{P_{i}\right\}$ with the simple divisor $D=\sum P_{i}$ (the support is empty if $D=0$ ). If $D$ is simple, $X=Y-D$ is an algebraic complex curve.

Let $E$ be a holomorphic vector bundle on $Y$. Fix a simple divisor $D=\sum P_{i}$, let $E_{P}$ be the fiber of $E$ at $P \in Y$. Set $E_{i}=E_{P_{i}}$ if $P_{i} \in D$. We shall consider only the parabolic structures, which we call special, connected with the $S U(2)$-bundles. For the general case we suggest [8] and also [1].

DeFInITIOn 3.1.1. A special parabolic vector bundle is given by the data $\{E, \mathcal{P}\} \equiv$ $\left\{E, D(\mathcal{P}), W_{i}\right\}$, where:

a) $E$ is a rank 2 holomorphic vector bundle such that $\operatorname{det} E=\mathcal{O}_{Y}$;

b) $D(\mathcal{P})=\sum_{i} \alpha_{i} P_{i}$ is a real weight divisor where $0<\alpha_{i}<1$;

c) $W_{i}$ is a proper subspace of $E_{i}, 0 \subset W_{i} \subset E_{i}$, for every $i$.

We set $D_{\mathcal{P}}=\operatorname{supp} D(\mathcal{P})=\sum_{i} P_{i}$ and $d_{\mathcal{P}}=\sum_{i} \alpha_{i}$. The trivial parabolic structure on $E$ is given by the divisor $D=0$.

Since in our case the weight sequence at any point has just one element (see [1]), we have encoded this information into a real divisor. We would like to stress that this 
is possible only in the rank 2 case. Let $L \subset E$ be a holomorphic sub-line bundle. Let $L_{i} \subset E_{i}$ be the fiber of $L$ at $P_{i} \in D$. We set

$$
\left\{\begin{array}{lll}
\gamma_{i}=\alpha_{i} & \text { if } \quad L_{i}=W_{i} \\
\gamma_{i}=-\alpha_{i} & \text { if } \quad L_{i} \neq W_{i} .
\end{array}\right.
$$

and define the real divisor

$$
D(L, \mathcal{P})=\frac{1}{2} \sum_{i=1}^{n} \gamma_{i} P_{i}
$$

which has degree $\operatorname{deg} D(L, \mathcal{P})=\frac{1}{2} \sum_{i=1}^{n} \gamma_{i}$. We define the parabolic degree $\operatorname{par}(L)$ of $L$ by the formula

$$
\operatorname{par}(L)=\operatorname{deg} L+\operatorname{deg} D(L, \mathcal{P})
$$

We give the following:

Definition 3.1.2. A special parabolic bundle $\{E, \mathcal{P}\}$ is called stable (respectively semistable) if $\operatorname{par}(L)<0$ (resp. par $(L) \leq 0)$ for every holomorphic line bundle $L \subset E$.

REMARK 3.1.3. With the trivial parabolic structure on $E$, we have $\operatorname{par}(L)=$ $\operatorname{deg} L$. Since $\operatorname{det} E=\mathcal{O}_{Y}$, parabolic stability in this case coincides with usual stability, see [8].

We prove now a standard result we will use in section 4 . For any holomorphic vector bundle $\mathcal{K}$ on $Y$ we set $h^{i}(\mathcal{K})=\operatorname{dim} H^{i}(Y, \mathcal{K})$.

Proposition 3.1.4. Let $\{E, \mathcal{P}\}$ be a special stable parabolic bundle and $L$ be a line bundle of degree $d$.

1. If $d \geq \frac{1}{2} d_{\mathcal{P}}+2 g-1$, then $h^{1}(E(L))=0$.

2. If $d \geq \frac{1}{2} d_{\mathcal{P}}+2 g$, then $E(L)$ is generated by its global sections.

Proof.

1. By Serre duality we have to prove that $h^{0}\left(E\left(L^{-1}\right) \otimes \omega_{Y}\right)=0$. Note that $\operatorname{det} E=\mathcal{O}_{Y}$ and hence that $E$ is self-dual. Assume by contradiction that $s \neq 0$ is a global section of $E\left(L^{-1}\right) \otimes \omega_{Y}$. It defines a map $L \otimes \omega_{Y}^{-1} \rightarrow E$. The parabolic degree of the image line bundle is bigger than $\operatorname{deg} L-\operatorname{deg} \omega_{Y}-$ $\frac{1}{2} \operatorname{deg} D(L, \mathcal{P}) \geq 0$. This gives a contradiction.

2. Fix a point $P \in Y$. Consider the exact sequence $0 \rightarrow E(L(-P)) \rightarrow E(L) \rightarrow$ $E(L)_{P} \rightarrow 0$. We have $H^{1}(E(L(-P)))=0$ by the first part of the proof. Hence the map $H^{0}(Y, E(L)) \rightarrow E(L)_{P}$ is surjective.

3.2. Flat bundles and parabolic structures. Let $X=Y-D$, where $Y$ is compact and $D=\sum P_{i}$ is simple. Let $\{F, \nabla\}$ be an $S U(2)$ flat vector bundle on $X$,

$$
\nabla: F \rightarrow F \otimes \omega_{X}
$$

is flat: $\nabla^{2}=0$ and there is an hermitian form $<,>$, on $F$ compatible with $\nabla$. We use the local construction described in section 2. Take a coordinate disk $U_{j}$ around a point $P_{j}$, then consider $U^{*}=U_{j}-\left\{P_{j}\right\}$. The restriction of $\{F, \nabla\}$ to $U_{j}^{*}$ extends (see 
2.1.3) to a singular connection defined on $U_{j}$. Repeating this construction at every point of $D$, we see there is an extension

$$
(E, \bar{\nabla})
$$

where $E$ is a holomorphic vector bundle on $Y,\left.E\right|_{X}=F$,

$$
\bar{\nabla}: E \rightarrow E(D) \otimes \omega_{Y}=E(D) \otimes \omega_{Y} .
$$

If $E_{j}$ is the fiber over $P_{j} \in D$, the residues define maps:

$$
\Gamma_{j}=\operatorname{Res}_{j}(\nabla): E_{j} \rightarrow E_{j},
$$

and (up to conjugation) $\exp \left(-\pi i \Gamma_{j}\right)$ gives the holonomy of $\nabla$ around $P_{j}$. The eigenvalues $\alpha_{j}$ and $-\alpha_{j}$ of $\Gamma_{j}$ are real with $0 \leq \alpha_{j}<1$. We define the weight divisor

$$
\sum_{i} \alpha_{j} P_{j}
$$

To define the parabolic structure (see 3.1.1) we set, for $\alpha_{j}>0$,

$$
W_{i}=\operatorname{ker}\left(\Gamma_{i}-\alpha_{i} i d\right) \text {. }
$$

Since $F$ is a $S U(2)$ flat vector bundle, $\operatorname{det} F$ is trivial and $\wedge^{2} \nabla=d$ is the trivial connection. Arguing as in 2.1.2 we see $\wedge^{2} \bar{\nabla}$ extends $\wedge^{2} \nabla$ and gives a regular connections on $\operatorname{det} E$, that is, $\left\{\operatorname{det} E, \wedge^{2} \bar{\nabla}\right\}$ is a flat unitary line-bundle with trivial monodromy: $\operatorname{det} E=\mathcal{O}_{Y}$.

Definition 3.2.1. When $\{E, \mathcal{P}\}=\left\{\sum \alpha_{i} P_{i}, W_{i}\right\}$ is the special parabolic vector bundle associated to $\{F, \nabla\}$ we write

$$
\tau(\{F, \nabla\})=\{E, \mathcal{P}\} .
$$

It follows that $\{E, \mathcal{P}\}=\tau(\{F, \nabla\})$ is semistable, and stable if the representation is irreducible. This gives an almost invertible functor:

TheOREM 3.2.2. The correspondence

$$
\{F, \nabla\} \mapsto \tau(\{F, \nabla\})=\{E, \mathcal{P}\}
$$

defines a one-to one functor between special stable parabolic bundles and irreducible $S U(2)$-bundles. Moreover, if $\{E, \mathcal{P}\}$ is semistable parabolic, there is an $S U(2)$-bundle $\{F, \nabla\}$ such that $\tau(\{F, \nabla\})=\{E, \mathcal{P}\}$.

Proof. See [8] and the appendix of [1].

We recall now a basic result we wiil need in section 4 .

LEMma 3.2.3. Let $s$ and $t$ be linearly independent meromorphic global sections of an irreducible $S U(2)$-bundle $\{E, \bar{\nabla}\}$ (i.e. the associated $\{E, \mathcal{P}\}$ is stable). If $s \wedge t=0$, then $\bar{\nabla} s \wedge \bar{\nabla} t \neq 0$.

Proof. The proof is standard. We assume $\bar{\nabla} s \wedge \bar{\nabla} t=0$ and we show that $\{E, \bar{\nabla}\}$ is reducible. First, since $s \wedge t=0$, we find a meromorphic function $g$ such that $t=g s$. It follows that $g$ is non-constant since $s$ and $t$ are linearly independent. Then one has:

$$
0=\bar{\nabla} s \wedge \bar{\nabla} t=\bar{\nabla} s \wedge \bar{\nabla}(g \cdot s)=(d g) s \wedge \bar{\nabla} s+g \cdot \bar{\nabla} s \wedge \bar{\nabla} s=d g \cdot(s \wedge \bar{\nabla} s),
$$


and hence

$$
s \wedge \bar{\nabla} s=0
$$

because $g$ is non constant. We obtain that the sub-bundle generated by $s$ is holonomyinvariant. Then $\{E, \bar{\nabla}\}$ is reducible. $\square$

3.3. Algebraic cmc-1 surfaces in $\mathcal{H}^{3}$. Fix a special semistable parabolic vector bundle $\{E, \mathcal{P}\}$ where $\mathcal{P}=\left\{D(\mathcal{P}), W_{i}\right\}, D(\mathcal{P})=\sum \alpha_{i} P_{i}$, and $D_{\mathcal{P}}=\operatorname{supp}(D(\mathcal{P})=$ $\sum_{i} P_{i}$. Let $\bar{\nabla}: E \rightarrow E(D) \otimes \omega_{Y}$ be the singular connection (see 3.2.2) whose restriction to $Y^{\prime}=Y \backslash D(\mathcal{P})$ gives an $S U(2)$ flat connection $\nabla$ on $F=\left.E\right|_{Y^{\prime}}$. We recall that $\operatorname{det} E=\mathcal{O}_{Y}$.

Definition 3.3.1. Two meromorphic sections $(s, t)$ of $E$ define a special meromorphic frame of $E$ if $s \wedge t \equiv 1$.

The meromorphic special frame $(s, t)$ of $E$ provides a holomorphic section of $E^{2} \otimes \mathcal{O}_{Y}(S)$ for a suitable divisor $S$. We can write:

$$
1=s \wedge t \in H^{0}\left(Y, \operatorname{det}\left(E^{2} \otimes \mathcal{O}_{Y}(S)\right)\right)=H^{0}\left(Y, \mathcal{O}_{Y}(2 S)\right) .
$$

Let $L \equiv O_{Y}(S)$ be the line bundle associated to $S$ and $\sigma \in H^{0}(Y, L)$ be the section corresponding to 1 . This means that the zero divisor of $\sigma: \mathcal{O}_{Y} \rightarrow L$ is $S$. Set $v=\sigma \cdot s$ and $w=\sigma \cdot t \in H^{0}(Y, E(L))$. The condition $s \wedge t=1$ becomes:

$$
v \wedge w=\sigma^{2} \in H^{0}\left(Y, L^{2}\right) ; \sigma \neq 0 .
$$

If conversely $L$ is a fixed line bundle and $v$ and $w$ in $H^{0}(Y, E(L))$ such that $v \wedge w=$ $\sigma^{2} \in H^{0}\left(Y, L^{2}\right)$ where $0 \neq \sigma \in H^{0}(Y, L)$ then $s=\frac{v}{\sigma}$ and $t=\frac{w}{\sigma}$ define a special meromorphic frame of $E$.

Definition 3.3.2. A pair of sections $(v, w)$ in $H^{0}(Y, E(L))$ defines a special frame of $E(L)$ if $0 \neq v \wedge w=\sigma^{2}, \sigma \in H^{0}(Y, L)$.

Let $D$ be a simple divisor and $X=Y \backslash D$.

Definition 3.3.3. A h-liftable map $f: X \rightarrow \mathcal{H}^{3}$ will be said to be of finite type if it is associated to a special meromorphic frame $(s, t)$ of a parabolic bundle $\{E, \mathcal{P}\}$.

To a special meromorphic frame $(s, t)$, we have associated in (9) a meromorphic quadratic differential $\Omega(s, t)=\bar{\nabla} s \wedge \bar{\nabla} t$ on $Y$. We have

Lemma 3.3.4. Let $(s, t)$ be a special meromorphic frame with poles on $S$. Then $\Omega(s, t)$ has poles on $2 S+D_{\mathcal{P}}$, that is: $\Omega(s, t) \in H^{0}\left(Y, \omega_{Y}^{2}\left(2 S+D_{\mathcal{P}}\right)\right)$.

Proof. It follows from lemma 2.2.2.

The meaning of the above lemma is the following: the condition $\operatorname{det}(s, t) \equiv 1$ forces the quadratic differential $\Omega(s, t)$ to have slightly milder singularities. The homogeneous form of the above quadratic differential is

$$
\Theta(v, w)=\sigma^{2} \cdot \Omega\left(\frac{v}{\sigma}, \frac{w}{\sigma}\right) \in H^{0}\left(Y, \omega_{Y}^{2} \otimes L^{2}\left(D_{\mathcal{P}}\right)\right),
$$

$v$ and $w$ in $H^{0}(Y, E(L))$ and $v \wedge w=\sigma^{2}$. We consider the case $\Omega(s, t)=0$, i.e. $\Theta(v, w)=0($ see 1.4.1).

DEFINITION 3.3.5. With the above notation 
1. a meromorphic special frame $(s, t)$ of $E$ will be said to be a meromorphic $\mathcal{B}$-frame of $E$ if $\Omega(s, t)=0$;

2. a special frame $(v, w)$ of $E(L)$ will be called a $\mathcal{B}$-frame of $E(L)$ if $\Theta(v, w)=0$;

3. a h-liftable non-constant map $f: X \rightarrow \mathcal{H}^{3}$ associated to a meromorphic $\mathcal{B}$ frame will be called an algebraic cmc-1 curve of hyperbolic space.

LEMma 3.3.6. Let $(s, t)$ be a meromorphic $\mathcal{B}$-frame of $E$ and $S$ be its polar divisor. Then $D=\operatorname{supp}(S)$ contains the support of the parabolic divisor: $D-D_{\mathcal{P}} \geq 0$ i.e. $D-D_{\mathcal{P}}$ is effective.

Proof. Use formula 14 .

4. Existence results. We fix a compact Riemann surface $Y$ of genus $g$ and a semistable parabolic bundle $\{E, \mathcal{P}\}$ on $Y$. The singular connection on $E$ will be $\bar{\nabla}$, its support divisor $D_{\mathcal{P}}$ and $d_{\mathcal{P}}=\operatorname{deg} D_{\mathcal{P}}$ (3.1.1).

4.1. The variety of special frames. Let $L$ be a holomorphic line bundle on $Y$ of degree $d$.

Hypothesis 4.1.1. From now on we assume $d \geq \frac{1}{2} d_{\mathcal{P}}+2 g$. Riemann-Roch and 3.1 .4 imply:

a) $h^{0}(L)=d-(g-1) ; h^{0}\left(L^{2}\right)=2 d-(g-1)$.

b) $h^{1}(E(L))=0$, that is, $h^{0}(E(L))=2 d-2(g-1)$.

c) $E(L)$ is generated by its global sections.

We define the basic determinant map $\phi: H^{0}(Y, E(L)) \times H^{0}(Y, E(L)) \rightarrow H^{0}\left(Y, L^{2}\right)$

$$
\phi\left(\omega_{1}, \omega_{2}\right)=\omega_{1} \wedge \omega_{2} .
$$

Let

$$
\left.\widetilde{Q}=\left\{\vartheta \in H^{0}\left(Y, L^{2}\right): \vartheta=\sigma^{2}: \sigma \in H^{0}(X, L)\right), \sigma \neq 0\right\} .
$$

Let $\mathbb{P}=\mathbb{P}\left(H^{0}\left(X, L^{2}\right)\right)$ be the projective space of $H^{0}\left(X, L^{2}\right)$. The locus $\widetilde{Q}$ is a homogenous cone, we let $\mathbb{Q}$ be its associated projective locus:

$$
\mathbb{Q}=\{(\vartheta): \vartheta \in \widetilde{Q}\} .
$$

The Veronese embedding $(\sigma) \rightarrow\left(\sigma^{2}\right)$ identifies $\mathbb{Q}$ and $\mathbb{P}\left(H^{0}(X, L)\right)$, the projective space associated to $H^{0}(X, L)$. In particular $\operatorname{dim} \mathbb{Q}=d-g$. We recall that $\left(\omega_{1}, \omega_{2}\right)$ gives rise to a special frame if and only if $\phi\left(\omega_{1}, \omega_{2}\right) \in \widetilde{Q}$. Let $\mathbb{G}=\mathbb{G}\left(2, H^{0}(Y, E(L))\right.$ be the Grassmannian of 2 -planes in $H^{0}(Y, E(L))$. We have $\operatorname{dim} \mathbb{G}=4(d-g)$.

Definition 4.1.2. The locus

$$
\mathcal{F}=\phi^{-1}(\widetilde{Q})
$$

will be called the locus of special frames of $E(L)$. A plane generated by a special frame, $\Pi=\operatorname{span}\left(\omega_{1}, \omega_{2}\right): \phi\left(\omega_{1}, \omega_{2}\right) \in \widetilde{Q}$, will be called special. Therefore the locus

$$
\mathcal{G}=\{\Pi \in \mathbb{G}: \Pi \text { is special }\}
$$

will be called the special planes locus. 
We remark that any basis of a special plane gives a special frame of $E(L)$. We have then a natural fibration $\psi: \mathcal{F} \rightarrow \mathcal{G}$ defined by:

$$
\psi\left(\omega_{1}, \omega_{2}\right)=\operatorname{span}\left(\omega_{1}, \omega_{2}\right) .
$$

Finally we define the mapping: $\varrho: \mathcal{G} \rightarrow \mathbb{Q} \subset \mathbb{P}$ by

$$
\varrho\left(\operatorname{span}\left(\omega_{1}, \omega_{2}\right)\right)=\left(\phi\left(\omega_{1}, \omega_{2}\right)\right)=\left(\omega_{1} \wedge \omega_{2}\right) .
$$

Let $\Upsilon \subset \mathbb{G} \times \mathbb{Q}$ be the closure of the graph $\Upsilon_{\varrho}$ of $\varrho$. That is :

$$
\Upsilon=\overline{\{(\Pi, \varrho(\Pi)): \Pi \in \mathcal{G}\}}=\bar{\Upsilon}_{\varrho} .
$$

Set $T=\mathbb{G} \times \mathbb{Q}$. We recall that using the Veronese embedding we have identified $\mathbb{Q}$ and $\mathbb{P}\left(H^{0}(Y, L)\right)$. Let $q_{1}: T \rightarrow \mathbb{G}$ and $q_{2}: T \rightarrow \mathbb{Q}$ be the projections. We still denote by $q_{1}: \Upsilon \rightarrow \mathbb{G}$ and $q_{2}: \Upsilon \rightarrow \mathbb{Q}$ the induced projection maps. We can identify $\mathcal{G}$ with $\Upsilon_{\varrho}$, and $q_{1} \mid \Upsilon_{\varrho}$ with $\varrho$. We have $q(\Upsilon)=\overline{\mathcal{G}}$ the closure of $\mathcal{G}$. Set $\Upsilon_{0}=\Upsilon \backslash \Upsilon_{\varrho}$, and $\mathcal{G}_{0}=\overline{\mathcal{G}}-\mathcal{G}$.

Proposition 4.1.3. Let $\Pi \in \mathcal{G}_{0}$ and $(v, w)$ be any basis of $\Pi$. Then $: v \wedge w=0$.

Proof. If $\Pi=\operatorname{span}(v, w) \in \Upsilon$ then its determinant $v \wedge w=\sigma^{2}$ is a square where $\sigma \in H^{0}(X, L) ; \sigma \neq 0$ if and only if $\Pi \in \mathcal{G}$. $\square$

4.2. Existence of special frames. We show under the hypothesis 4.1.1 that the locus of special frames of $E(L)$ is not empty. Let $v \in H^{0}(Y, E(L))$ be a fixed section. Let $\phi_{v}: H^{0}(Y, E(L)) \rightarrow H^{0}\left(X, L^{2}\right)$ be the linear map :

$$
\phi_{v}(\omega)=\phi(v, \omega)=v \wedge \omega
$$

We have a well-known:

LEMMA 4.2.1. If $v$ is a general section then $\operatorname{dim} \operatorname{ker}\left(\phi_{v}\right)=1$, i.e. $\operatorname{ker}\left(\phi_{v}\right)$ is generated by $v$.

Proof. Since $E(L)$ is generated by global section we can find $v \in H^{0}(Y, E(L))$ without zeros. Assume $v \wedge \omega=0$, then $\omega=f \cdot v$ where $f$ is a meromorphic function on $Y$. Since $v$ has no zeros, then $f$ has no poles, and so it is constant.

Denote by $\Phi_{v}=\{(a) \in \mathcal{P}, \quad a=v \wedge \omega\}$. It follows from lemma 4.2 .1 that $\Phi_{v}$ is a projective space of dimension $h^{0}(E(L))-2=2 d-2 g$, that is, $\Phi_{v}$ has codimension $g$ in $\mathbb{P}$. Since $\mathbb{Q}$ is a projective subvariety of codimension $g$ of $\mathbb{P}$, we have proved:

Proposition 4.2.2. The locus $\mathbb{Q}_{v}=\mathbb{Q} \cap \Phi_{v}$ is projective of codimension $e \leq d+g$. That is, $\operatorname{dim} \mathbb{Q}_{v} \geq d-2 g$ and, in particular, (by 4.1.1) it is not empty.

A dimension count gives:

COROLlary 4.2.3. The loci $\mathcal{F}$ and $\mathcal{G}$ are not empty and moreover:

$$
\operatorname{dim} \mathcal{F} \geq 3 d-(4 g-4), \operatorname{dim} \mathcal{G} \geq 3 d-4 g .
$$

Proof. The algebraic locus $\mathcal{F} \subset H^{0}(Y, E(L))^{2}$ is not empty by 4.2.2. Let $p_{i}$ : $H^{0}(Y, E(L))^{2} \rightarrow H^{0}(Y, E(L)), i=1,2$ be the projections. Then $p_{1}(\mathcal{F})$ contains the general point $v$ and so an open Zariski set. Finally $p_{2}\left(p_{1}^{-1}(v) \cup \mathcal{F}\right)$ is the locus

$$
\Gamma_{v}=\left\{w \in H^{0}(Y, E(L)):(v \wedge w)=\left(\phi_{v}(w)\right) \in \mathbb{Q}\right\}=\phi_{v}^{-1}\left(\mathbb{Q}_{v}\right) .
$$

Since (see 4.2.2) the codimension $\mathbb{Q}_{v}$ is $d$ we have dimension $\operatorname{dim} \Gamma_{v}=d-2 g+2$. We finally obtain $\operatorname{dim} \mathcal{F}=\operatorname{dim} \Gamma_{v}+h^{0}(E(L))=d-2 g+2+2 d-2 g+2=3 d-4 g+4$. 
4.3. The variety of $\mathcal{B}$-frames . Now we will study the loci defined by the Bryant condition (see 1.4.1). If $(v / \sigma, w / \sigma)$ is a special frame we defined (see 20) :

$$
\Theta(v, w)=\sigma^{2} \cdot \bar{\nabla}\left(\frac{v}{\sigma}\right) \wedge \bar{\nabla}\left(\frac{w}{\sigma}\right)
$$

where $v \wedge w=\sigma^{2}$ and $\left.0 \neq \sigma \in H^{0}(Y, L)\right)$. We also recall (see 4.1.2) that the locus of the special frames is $\mathcal{F}=\left\{(v, w) \in H^{0}(E(L)) \times H^{0}(E(L)): v \wedge w=\sigma^{2}, \sigma \in H^{0}(L), \sigma \neq 0\right\}$.

Since $\Theta(v, w)$ is invariant under the changing of sign in $\sigma$, it defines a map:

$$
\Theta: \mathcal{F} \rightarrow H^{0}\left(Y, \omega_{Y}^{2}\left(D_{\mathcal{P}}\right)\right) .
$$

The equation $\Theta(v, w)=0$ holds (see 3.3.5) for the $\mathcal{B}$-frame $(v, w)$ of $E(L)$. We also defined $\psi: \mathcal{F} \rightarrow \mathbb{G}, \psi\left(\omega_{1}, \omega_{2}\right)=\operatorname{span}\left(\omega_{1}, \omega_{2}\right)$. Accordingly we give the following:

Definition 4.3.1. A special plane $\Pi \in \mathcal{G} \subset \mathbb{G}$ will be called a $\mathcal{B}$ plane if it is generated by a $\mathcal{B}$-frame. We set:

a) $\mathcal{B}=\Theta^{-1}(0) \subset \mathcal{F} \equiv$ the locus of $\mathcal{B}$-frames.

b) $\mathcal{M}=\psi(\mathcal{B}) \subset \mathbb{G} \equiv$ the locus of $\mathcal{B}$-planes.

We remark that any basis of a $\mathcal{B}$-plane is a $\mathcal{B}$-frame of $E(L)$.

REMARK 4.3.2. The locus of the special planes $\mathcal{G}$ is not compact in general, and moreover (see 23) $\varrho: \mathcal{G} \rightarrow \mathbb{Q}$ does not extend to the closure $\overline{\mathcal{G}} \subset \mathbb{G}$. We notice however that if the condition $v \wedge w=\sigma^{2}$ is dropped formula (20) is well defined for $\sigma \neq 0$.

We defined $T=\mathbb{G} \times \mathbb{Q}$ with projections $q_{1}: T \rightarrow \mathbb{G}$ and $q_{2}: T \rightarrow \mathbb{Q}$. Let $S_{\mathbb{G}}$ be the tautological rank 2 vector bundle of $\mathbb{G}$, that is:

$$
S_{\mathbb{G}}=\left\{(\Pi, v) \in \mathbb{G} \times H^{0}(Y, E(L)): v \in \Pi\right\} .
$$

The line bundle $\wedge^{2} S_{\mathbb{G}}$ generates the Picard group of $\mathbb{G}$ ([5] chapter 2) and its dual is ample. Let $\mathcal{O}_{\mathbb{Q}}(-1)$ be the tautological line bundle of $\mathbb{Q}=\mathbb{P}\left(H^{0}(X, L)\right)$. The pull-backs: $M=q_{1}^{*}\left(\bigwedge^{2} S_{\mathbb{G}}\right)$ and $N=q_{2}^{*}\left(\mathcal{O}_{\mathbb{Q}}(-1)\right)$ are line bundles on $T$. Define the trivial bundles

$$
\mathbb{V}=H^{0}\left(Y, \omega_{Y}^{2} L^{4}\left(\mathcal{D}_{\mathcal{P}}\right)\right) \times T, \mathbb{W}=H^{0}\left(Y, \omega_{Y}^{2} L^{2}\left(\mathcal{D}_{\mathcal{P}}\right)\right) \times T .
$$

Given $v$ and $w$ in $H^{0}(Y, E(L))$ the formula

$$
\Psi(v, w, \sigma)=\sigma^{4} \bar{\nabla} \frac{v}{\sigma} \wedge \bar{\nabla} \frac{w}{\sigma}
$$

is well defined if $0 \neq \sigma \in H^{0}(X, L)$ and $\Psi(v, w, \sigma) \in \mathbb{V}$. Letting $\lambda \in \mathbb{C}, A \in G L(2, \mathbb{C})$, we observe the homogeneity:

$$
\Psi(A v, A w, \lambda \sigma)=\lambda^{4} \sigma^{4} \bar{\nabla} \frac{A v}{\lambda \sigma} \wedge \bar{\nabla} \frac{A w}{\lambda \sigma}=\operatorname{det} A \lambda^{2} \Psi(v, w, \sigma) .
$$

This defines a bundle map:

$$
\Psi: M \otimes N^{2} \rightarrow \mathbb{V} .
$$

Now we study the restriction of $\Psi$ to $\Upsilon$. First we see by 3.3 .4 that on $\Upsilon_{\varrho}$ we have

$$
\Psi(\sigma, v, w)=\sigma^{2} \Theta(v, w)
$$


where $\Theta(v, w) \in H^{0}\left(Y, \omega_{Y}^{2} L^{2}\left(\mathcal{D}_{\mathcal{P}}\right)\right)$. Then the locus

$$
\Gamma=\left\{(\operatorname{span}(v, w),(\sigma)) \in T: \Psi(\sigma, v, w)=\sigma^{2} \eta, \eta \in H^{0}\left(Y, \omega_{Y}^{2} L^{2}\left(\mathcal{D}_{\mathcal{P}}\right)\right)\right\}
$$

is closed and $\Upsilon_{\varrho} \subset \Gamma$, so that $\Upsilon \subset \Gamma$. In fact if $\Pi=\operatorname{span}(v, w) \in \Upsilon$ we have:

$$
\Psi(v, w, \sigma) \in \sigma^{2} \mathbb{W} \subset \mathbb{V} .
$$

This observation is essentially the content of 3.3.4. We will denote with $\mathbb{W}_{\Gamma}$ and $M_{\Gamma}$ the restriction of $\mathbb{W}$ and $M$ to $\Gamma$. Let, for $\sigma \neq 0$,

$$
\bar{\Psi}(v, w, \sigma)=\sigma^{-2} \Psi(v, w, \sigma) .
$$

We have the homogeneity:

$$
\bar{\Psi}(A v, A w, \lambda \sigma)=\operatorname{det}(A) \bar{\Psi}(v, w, \sigma) .
$$

Therefore the restriction of $\Psi$ to $\Gamma$ defines a bundle map $\bar{\Lambda}: M_{\Gamma} \rightarrow \mathbb{W}_{\Gamma}$, and this bundle map is well defined on $\Upsilon \subset \Gamma$. Let $\left.M\right|_{\Upsilon}=\widetilde{M}$ be the restriction of $M$ to $\Upsilon$ and denote for sake of notation by $\mathbb{W}_{\Upsilon}$ the restriction of $\mathbb{W}$ to $\Upsilon$. It follows that the restriction of $\Psi$ to $\Upsilon$ defines a vector bundle map:

$$
\Lambda: \widetilde{M} \rightarrow \mathbb{W}_{\Upsilon}
$$

In the case of irreducible monodromy the boundary loci $\mathcal{G}_{0}$ and $\Upsilon_{0}$ do not intersect the zeroes of $\Lambda$. In fact we have the following:

Lemma 4.3.3. Assume that $E$ is (parabolic) stable. Then $Z_{\Lambda} \cap \Upsilon_{0}=\emptyset$. In particular the projection $q_{1}=\varrho: \Upsilon_{\rho} \rightarrow \mathbb{G}$ defines an isomorphism between $Z_{\Lambda}$ and the locus $\mathcal{M}$ of $\mathcal{B}$ planes.

Proof. It is the content of lemma 3.2.3. $\mathrm{\square}$

Now we state our existence result:

TheOREM 4.3.4. Assume that $\{E, \bar{\nabla}\}$, is associated to an irreducible $S U(2)$-flat representation, i.e., $\{E, \mathcal{P}\}$ is stable. We set $\operatorname{deg} D_{\mathcal{P}}=d_{\mathcal{P}}$. Let $L$ be a holomorphic line-bundle of degree $\operatorname{deg} L=d$. Suppose

$$
d \geq 7 g-3+d_{\mathcal{P}} .
$$

Then the locus $\mathcal{M}$ of $\mathcal{B}$-planes of $E(L)$ is a nonempty algebraic set of dimension $a \geq d-7(g-1)-d_{\mathcal{P}}-4$.

Proof. By 4.3.3 we have to show that $Z_{\Lambda}$ is not empty of dimension $a \geq d-7(g-$ $1)-d_{\mathcal{P}}$. We consider $\Lambda \in H^{0}\left(\mathbb{W}_{\Upsilon} \otimes(\widetilde{M})^{-1}\right)$. Since by $4.2 .3 \operatorname{dim} \Upsilon=\operatorname{dim} \mathcal{G} \geq 3 d-4 g$ it is enough to show the top Chern class $c_{\text {top }}$ of $\mathbb{W}_{\Upsilon} \otimes(\widetilde{M})^{-1}$ is not zero. Since $\mathbb{W}_{\Upsilon}$ is trivial we have

$$
c_{t o p}=c_{1}\left(\widetilde{M}^{-1}\right)^{r},
$$

where $r=2 d+3 g-3+d_{\mathcal{P}}$ is the rank of $\mathbb{W}_{\Upsilon}$. Let $i: \Upsilon \hookrightarrow \mathbb{G}$ be the inclusion. It follows then

$$
c_{\text {top }}=i^{*}\left(q_{1}^{*}(h)^{r}\right),
$$


where $h=c_{1}\left(\wedge^{2} S_{\mathbb{G}}\right)^{*}$ is positive since $\wedge^{2} S_{\mathbb{G}}^{*}$ is ample. We compute the cohomology class $\mu=q_{1 *} i_{*}\left(c_{\text {top }}\right)$ of $\mathbb{G}$. First we obtain:

$$
i_{*} c_{\text {top }}=i_{*} i^{*}\left(q_{1}^{*}(h)^{r}\right)=q_{1}^{*}\left(h^{r}\right) \cdot[\Upsilon],
$$

where $[\Upsilon]$ denotes the class of the variety $\Upsilon \subset T$. Similarly we let $[\overline{\mathcal{G}}]$ be the class of $\overline{\mathcal{G}}$ in $G$. Then we get

$$
\mu=q_{1 *} q_{1}^{*}\left(h^{r}\right) \cdot[\Upsilon]=h^{r} \cdot q_{1 *}[\Upsilon]=h^{r} \cdot[\overline{\mathcal{G}}] \neq 0
$$

since $h$ is positive and the dimension of $\overline{\mathcal{G}}$ is larger than $r$. It follows that $c_{\text {top }} \neq 0$, which proves the theorem.

Proof of the Existence Theorem. One observes that 4.3.4 provides $\mathcal{B}$-meromorphic frames of $E$ in the case of any irreducible monodromy on $Y-D$. If $(s, t)$ is such a $\mathcal{B}$-frame, let $P_{s}$ and $P_{t}$ be the polar divisors of $s$ and $t$ respectively. Let $Z=\{z \in Y$ : $\nabla s(z)=\nabla t(z)=0\}$ the branch points divisor. Let $H=P_{s} \cup P_{t} \cup Z$ be the union. We define a cmc-1 immersion (see 1.4.3) $f: Y \backslash(D \cup H) \rightarrow \mathcal{H}^{3}$. By construction the monodromy of $f$ is the monodromy of $E$.

5. Higgs fields and the period problem. In this section we use the special frames to obtain a standard form for the flat connection. Then the period problem is translated into a holonomy problem. The language of Higgs fields (see [7] and [12]) allows, in particular, to discuss the condition given in [10].

5.1. Higgs fields associated to h-liftable map. Let $G=S L(n, \mathbb{C})$ and let $H$ be a closed subgroup of $G$. Let $H_{e}$ be the connected component of $H$ containing $e$ : we assume $H_{e}=S U(n)$. Let $f: X \rightarrow S L(n) / H$ be h-liftable, $\{F, \nabla\}$ be the associated flat bundle. The associated special frame, $e_{1}, \ldots, e_{n}$, gives a holomorphic trivialization of $F$ :

$$
F \equiv{ }_{f} \mathcal{O}_{X}^{n}
$$

Since $f$ is $h$-liftable, we have $\nabla e_{i} \in H^{0}\left(X, \omega_{X}\right)^{n}$. We write

$$
\nabla e_{i}=\left(\omega_{1, i}, \ldots \omega_{n, i}\right) .
$$

Transposing we obtain a matrix of 1.0-forms.

$$
\Theta={ }^{t}\left(\nabla e_{1}, \ldots, \nabla e_{n}\right)=\left(\omega_{i, j}\right)
$$

We will identify $\Theta$ with the associated map $\Theta: F \rightarrow F \otimes \omega_{X}$, that is $\Theta \in$ $H^{0}\left(X, H o m\left(F, F \otimes \omega_{X}\right)\right)$. We may write

$$
\nabla=d+\Theta
$$

The decomposition $\nabla=D^{\prime}+D^{\prime \prime}$ gives $D^{\prime}=\partial+\Theta$ and $D^{\prime \prime}=\bar{\partial}$. The condition that $\nabla$ is special translates into the vanishing of the trace of $\Theta$. In fact one has

$$
0=\left(\wedge^{n} \nabla\right)\left(e_{1} \wedge \ldots \wedge e_{n}\right)=\left(\sum_{i=1}^{n} \omega_{i, i}\right) e_{1} \wedge \ldots \wedge e_{n},
$$

and hence

$$
\sum_{i=1}^{n} \omega_{i, i}=0
$$


It follows that $\Theta$ is a $\operatorname{sl}(n, \mathbb{C})$ matrix.

DeFinition 5.1.1. The matrix $\Theta$ in (29) will be called the Higgs field associated to $f$.

Let $M_{\Theta} \subset S L(n, \mathbb{C})$ be the holonomy group of $\nabla$. The topological closure $K_{\Theta}=$ $\overline{M_{\Theta}}$ of $M_{\Theta}$ in $S L(n, \mathbb{C})$ (by Cartan theorem) defines a Lie-subgroup. By construction one has that the connected component of $K_{\Theta}$ through $e$ is contained in $H=S U(n)$.

Conversely given on $F=\mathcal{O}_{X}^{n}$ a connection $\nabla=d+\Theta$ where $\Theta=\left(\omega_{i, j}\right)$ is a tensor of type $(1,0)$, we have:

1. $\nabla^{2}=0$ if and only if $\bar{\partial}(\Theta)=0$ (i.e. the $\omega_{i, j}$ are holomorphic).

2. $\nabla$ is a $S L(n, \mathbb{C})$ connection if and only if the trace of $\Theta$ vanishes, i.e. $\sum_{i=1}^{n} \omega_{i, i}=0$.

Given a holomorphic trace-free Higgs field $\Theta$, let $\nabla_{\Theta}=d+\Theta$ be the associated connection. Let $M_{\Theta}$ be its holonomy group and $K_{\Theta}$ be its closure in $G$. We give the following:

DEFINITION 5.1.2. (Non abelian period problem): We say that $\Theta$ satisfies the period problem if the connected component of $K_{\Theta}$ through e is contained in $S U(n)$.

We may state:

Proposition 5.1.3. Assume $\Theta$ satisfies the period problem of 5.1.2. Then the standard basis of $\mathcal{O}_{X}^{n}$ defines a h-liftable map $f: X \rightarrow G / H$ where $H \supset K_{\Theta}$ and $H_{e}=S U(n)$.

Proof. For any simply connected open set $U$ of $X$ fix flat unitary basis $f_{1}, \ldots f_{n}$ of $\mathbb{C}^{n}$ consider the matrix $A=\left(a_{i, j}\right)$ such that $e_{i}=\sum_{j} a_{i, j} f_{j}$. Then define $f(x)=$ $A(x) \cdot A^{*}(x) \bmod H$ for $x \in U$. $\mathrm{u}$

REMARK 5.1.4. It appears difficult to provide conditions in order to solve the above period problem.

5.2. Higgs fields and Maurer-Cartan form. We consider again a $h$-liftable map $f: X \rightarrow G / H$ where $G=S L(n, \mathbb{C})$ and $H_{e}=S U(n)$ as in the previous section. We let $\Theta$ be the Higgs field associated to $f$ (see 5.1.1) and $\nabla=d+\Theta$ be the associated flat connection on $F=\mathbb{C}^{n}$. Let $\{U, z\}$ be a simply connected coordinate open set. Using the canonical frame of $\mathbb{C}^{n}$, we set $E=\left(e_{1}, \ldots, e_{n}\right)$. As in proposition 5.1.3, we have matrices $A(z)$ such that

$$
f(x)=A(z(x)) \cdot A^{*}(z(x)) \quad \bmod H
$$

for $x \in U$. Moreover $A^{-1} E=R=\left(f_{1}, \ldots f_{n}\right)$ is a unitary frame of $\left.F\right|_{U}$. Taking derivative in the equation $A \cdot R=E$ we find

$$
d A \cdot R=\nabla(A \cdot R)=\nabla E=\theta \cdot E=\theta A \cdot R
$$

that is:

$$
\Theta=d A \cdot A^{-1} .
$$

Now comparing with $[10$, section 3] we see that $\Theta$ is pull-back to $U$ of the right invariant Maurer- Cartan form of $G=S L(n, \mathbb{C})$, that is:

Proposition 5.2.1. The Higgs field $\Theta$ is the pull-back of the right-invariant Maurer Cartan form to $X$. 
If we now consider the matrix of quadratic forms:

$$
\Theta^{2}=\left(\beta_{i, j}\right)=\sum_{k=1}^{n} \omega_{i, k} \omega_{k . j}
$$

we have the following generalization of the Bryant condition:

Kokubu-Takahashi-Umehara-Yamada condition (see [10, 3.3])

$$
\operatorname{trace}\left(\Theta^{2}\right)=\sum_{k=1}^{n} \beta_{k, k}=\sum_{i, k} \omega_{i, k} \omega_{k, i}=0 .
$$

We specialize formula (31) to the case $n=2$ to get the Bryant condition (1.4.1), that is $\nabla e_{1} \wedge \nabla e_{2}=0$. If we write the $s l(2)$ matrix:

$$
\Theta=\left(\begin{array}{cc}
\alpha & \gamma \\
\beta & -\alpha
\end{array}\right)
$$

we obtain $\nabla e_{1}=\alpha e_{1}+\beta e_{2}$ and $\nabla e_{1}=\gamma e_{1}-\alpha e_{2}$, and hence

$$
\nabla e_{1} \wedge \nabla e_{2}=-\alpha^{2}-\beta \gamma=\operatorname{det} \Theta
$$

Then the frame satisfies the Bryant condition if and only if $\operatorname{det} \Theta=0$. That is $\Theta$ is nihilpotent: $\Theta^{2}=\operatorname{det} \Theta \mathrm{I}$ or equivalently the trace of $\Theta^{2}$ vanishes.

REMARK 5.2.2. Cousin's representation of a (local) minimal surface in Euclidean space is obtained, when $\operatorname{det} \Theta=0$, taking $\omega_{1}=\alpha, \omega_{2}=i(\beta+\gamma)$ and $\omega_{3}=i(\beta-\gamma)$.

\section{REFERENCES}

[1] P. Belkale, Local System on $\mathbb{P}^{1} \backslash S$ for $S$ a Finite Set, Compositio Math, 129 (2000), pp. $67-86$.

[2] R. BRyant, Surfaces of mean curvature one in hyperbolic space, Astérisque, Soc. Math de France, 154-155 (1987), pp. 321-347.

[3] L. DE Lima AND P. RoITMAn, Constant mean curvature one surfaces in hyperbolic 3-space using the Bianchi-Calò method, An. Acad. Brasil. Ciènc., 74 (2002), pp. 19-24.

[4] S. K. Donaldson, Boundary value problems for Yang-Mills fields, Jour. Geom. Phy., 8 (1992), pp. 89-122.

[5] P. Griffiths and J. Harris, Principles of Algebraic Geometry, Wiley-Interscience, New York, 1978.

[6] W. Meens, A. Ros And H. Rosenberg, The Global Theory of Minimal Surfaces in Flat Spaces, Lecture Notes in Math., 1775 Springer, Berlin-Heidelberg-New York.

[7] N. J. Hitchin, The self-duality equations on a Riemann surface, Proc. London Math. Soc. (3), 55 (1987), pp. 59-126.

[8] V. Mehta and C. Seshadri, Moduli of vector bundles with parabolic structures, Math. Ann., 248 (1980), pp. 205-239.

[9] S. Kobayashi, Differential Geometry of Complex Vector Bundles, Princeton University Press, Princeton, NJ; Iwanami Shoten, Tokyo, 1987.

[10] M. Kokubu, M. Takahashi, M. Umehara, and K. Yamada, An analogue of minimal surface theory in $\mathrm{SL}(n, \mathbf{C}) / \mathrm{SU}(n)$, Trans. Amer. Math. Soc., 354 (2002), pp. 1299-1325.

[11] G. PIrola, Algebraic curves and non rigid minimal surfaces in the euclidean space, Pacific J. Math., 183 (1998), pp. 333-357.

[12] C. Simpson, Higgs bundles and local system, Inst. Hautes Ètudes Sci. Publ. Math., 75 (1992), pp. 5-95.

[13] A. J. Small, Surfaces of constant mean curvature 1 in $H^{3}$ and algebraic curves on a quadric, Proc. Amer. Math. Soc., 122 (1994), pp. 1211-1220.

[14] M. Umehara And K. Yamada, Complete surfaces of constant mean curvature 1 in the hyperbolic 3-space, Annals of Math., 137 (1993), pp. 611-638. 
G. P. PIROLA 